\title{
Multifaceted effects of synthetic TLR2 ligand and Legionella pneumophilia on Treg-mediated suppression of $\mathrm{T}$ cell activation
}

\author{
Wendy WC van Maren', Stefan Nierkens', Liza W Toonen ${ }^{1}$, Judith M Bolscher ${ }^{2}$, Roger PM Sutmuller ${ }^{1,2+}$ and \\ Gosse J Adema ${ }^{1 *+}$
}

\begin{abstract}
Background: Regulatory T cells (Treg) play a crucial role in maintaining immune homeostasis and self-tolerance. The immune suppressive effects of Tregs should however be limited in case effective immunity is required against pathogens or cancer cells. We previously found that the Toll-like receptor 2 (TLR2) agonist, Pam3CysSK4, directly stimulated Tregs to expand and temporarily abrogate their suppressive capabilities. In this study, we evaluate the effect of Pam3CysSK4 and Legionella pneumophila, a natural TLR2 containing infectious agent, on effector T (Teff) cells and dendritic cells (DCs) individually and in co-cultures with Tregs.

Results: TLR2 agonists can directly provide a co-stimulatory signal inducing enhanced proliferation and cytokine production of naive CD4+ Teff cells. With respect to cytokine production, DCs appear to be most sensitive to low amounts of TLR agonists. Using wild type and TLR2-deficient cells in Treg suppression assays, we accordingly show that all cells (e.g. Treg, Teff cells and DCs) contributed to overcome Treg-mediated suppression of Teff cell proliferation. Furthermore, while TLR2-stimulated Tregs readily lost their ability to suppress Teff cell proliferation, cytokine production by Teff cells was still suppressed. Similar results were obtained upon stimulation with TLR2 ligand containing bacteria, Legionella pneumophila.
\end{abstract}

Conclusions: These findings indicate that both synthetic and natural TLR2 agonists affect DCs, Teff cells and Treg directly, resulting in multi-modal modulation of Treg-mediated suppression of Teff cells. Moreover, Treg-mediated suppression of Teff cell proliferation is functionally distinct from suppression of cytokine secretion.

\section{Background}

The immune system is of crucial importance to our health and survival. Faced with pathogenic threats from outside as well as the rise of cancer cells from within, our immune defense must be able to cope with very diverse opponents. Mammals have developed a diverse set of receptors that sense components derived from pathogens and damaged cells. Amongst the best studied receptors are the so called pattern recognition receptors (PRR) like the Toll-like receptor (TLR) family, RIG-Ilike receptor (RLR) family and the NOD-like receptor

\footnotetext{
* Correspondence: G.Adema@ncmls.ru.nl

+ Contributed equally

'Department of Tumor Immunology, Nijmegen Centre for Molecular Life Sciences, Radboud University Nijmegen Medical Centre, Nijmegen, the Netherlands

Full list of author information is available at the end of the article
}

(NLR) family of proteins [1]. In general, engagement of these receptors on immune cells results in their activation, like enhanced antigen presentation, inflammatory cytokine production and the acquisition of immune effector function [2].

Pathogen recognition through specific TLRs can be of crucial importance for the induction of protective immunity. For instance, TLR4-deficient mice are more susceptible for infections with Neisseria meningitidis, E. coli, Haemophilus influenzae, Salmonella enteritidis, and Klebsiella pneumonia [3]. In this regard, the immunological effects of TLR2 ligation are remarkably different compared to the other TLRs (reviewed by Netea et al [3]). Firstly, TLR2 has been reported to direct the broadest repertoire of danger-associated molecular patterns from a large variety of pathogens, including grampositive and gram-negative bacteria, fungi, viruses, and

\section{Ciomed Central}


parasites, but also endogenous proteins like Heat Shock Protein 60 (HSP60) [4]. This broad range of recognition may be explained by the heterodimerization of TLR2 with either TLR1 or TLR6. However, the recent publication of the TLR1/2 receptor-ligand crystal structure [5], in combination with the extremely high affinity of TLR2 for its lipoprotein ligands [6], increases the possibility that a number of putative TLR2-ligands have no intrinsic TLR2-activating capacities but were actually contaminated by lipoproteins [6]. Secondly, TLR2-deficient mice are less susceptible to lethal infections with Aspergillus fumigatus, Yersinia enterocolitica or Candida albicans, which is in contrast with e.g. TLR4-deficient mice [7]. In TLR2-deficient mice, resistance to C. albicans is mediated by a stronger Th1 response due to diminished production of IL-10 during the infectious challenge [8]. The distinct roles of TLR2 and TLR4 in immunomodulation was further emphasized by findings that TLR2deficient mice experienced increased joint inflammation in preclinical rheumatoid arthritis (RA) models, while TLR4-deficient mice were more resistant [9]. Interestingly, the enhanced immunological responses in TLR2deficient mice correlate with decreased numbers of Tregs in these mice [8]. Moreover, C. albicans induced proliferation and survival of Tregs in a TLR2-dependent manner [8].

Different types of Tregs have been characterized and these Tregs are indispensable for the maintenance of immunologic self-tolerance and immune homeostasis [10]. The naturally occurring CD25+CD4+FoxP3+ Tregs are generated in the thymus and constitute about $5-15 \%$ of the peripheral CD4+ T cells in healthy animals and humans [11-13]. Once naturally occurring Tregs are activated via TCR-triggering, they are able to actively suppress the function of multiple immune cells, such as CD4+CD25-effector T cells (Teffs) and antigen presenting cells (APCs). Although these activities are essential for maintaining tolerance and preventing autoimmunity, their suppressive capacity may interfere with the development of a potential anti-tumor/anti-pathogen immune response, implicating the need for a mechanism that regulates Tregs. We recently demonstrated that TLR2 triggering on Tregs by Pam3Cys in combination with $\mathrm{T}$ cell receptor (TCR) activation resulted in proliferation of the otherwise non-proliferating Tregs and, importantly, the temporal abrogation of their suppressive capabilities [14]. After a resting period, the Tregs regain their suppressive, non-proliferative phenotype, indicating this is a reversible process.

Since TLR2 is widely expressed in immune cells, we now investigated the consequences of TLR2-signaling on different immune subsets involved in adaptive immune responses; APCs, Teffs and Tregs. We found increased TLR2 expression on activated immune cells.
Interestingly, although TLR2 triggering abrogated Tregmediated suppression of Teff cell proliferation, TLR2 triggering was unable to restore effector cytokine secretion. In addition, we show that Pam3Cys acts on Tregs, Teffs and APCs to reverse the suppressive effects of Tregs. Similar results were found with the pathogen Legionella pneumophila (HKLP) containing potent TLR2 ligands [15-17], emphasizing the crucial role of TLR2 in immunomodulation in infections.

\section{Results}

TLR2 expression and effects on Treg, Teffs and DCs

To decipher the role of TLR2 in immune responses in more detail, the expression and function of TLR2 on CD4+CD25+Foxp3+ Tregs, CD4+ Teffs and DCs were studied. Expression of TLR2 is readily detected on the cell surface of freshly isolated DCs (Figure 1A), while expression on freshly isolated CD4+ Teffs and Tregs is low or absent (Figure 1A). In line with the data from Liu et al. [18], TLR2 expression is up-regulated upon activation of these $\mathrm{T}$ cells (data not shown), and both CD4+ T cell populations continue to express TLR2 when cultured for several weeks with CD3 and Pam3Cys (Figure 1B).

Since the expression of TLR2 on these T cell subsets seems to be affected by their activation state, we investigated how TLR2 expression correlated to the functional responsiveness of DCs, Teffs and Tregs to the TLR2 ligand Pam3Cys. As shown in Figure 2, both freshly isolated Tregs and naive Teffs showed enhanced proliferation after CD3 stimulation in the presence of increasing amounts of TLR2-ligand, indicating Pam3Cys co-stimulated these $\mathrm{T}$ cells. In the absence of CD3 stimulation, no proliferation was observed on either Teffs or Tregs (data not shown), which is in line with the absence of TLR2 up-regulation on these cells. Analysis of the cultured CD4+ T cell subsets revealed that only the cultured Tregs, not the cultured Teff cells, remained responsive towards TLR 2 stimulation. This finding is consistent with our current understanding that primed Teff cells become less dependent on co-stimulatory signals, as has been described for CD28 stimulation.

TLR2-triggering was also highly effective in costimulating cytokine secretion in CD3-activated, freshly isolated Teff cells (Figure 3 ). They predominantly produced IL-2, IL-10, IL-4 and IFN $\gamma$, and cytokine production was gradually increased with increasing Pam3Cys concentrations. As expected, freshly isolated Tregs did not produce any detectable levels of these cytokines (data not shown). Also the in vitro expanded Tregs did not produce any cytokines while the in vitro cultured Teff cells (Figure 3) already produced high amounts of cytokines in the absence of Pam3Cys (i.e. only in the presence of anti-CD3). Adding increasing amounts of 


\section{A Fresh spleen-isolated naive DCs (CD11c $)$ Fresh spleen-isolated naive Tregs}

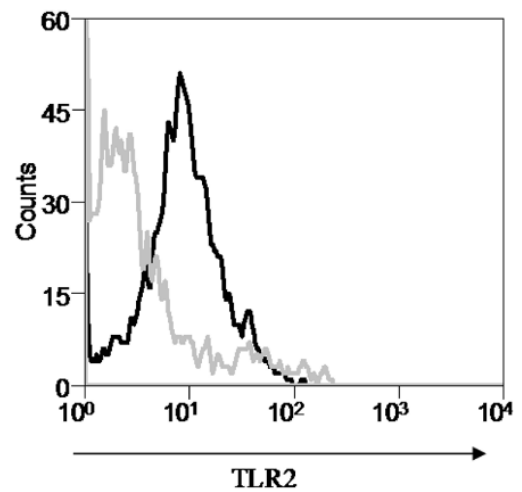

B
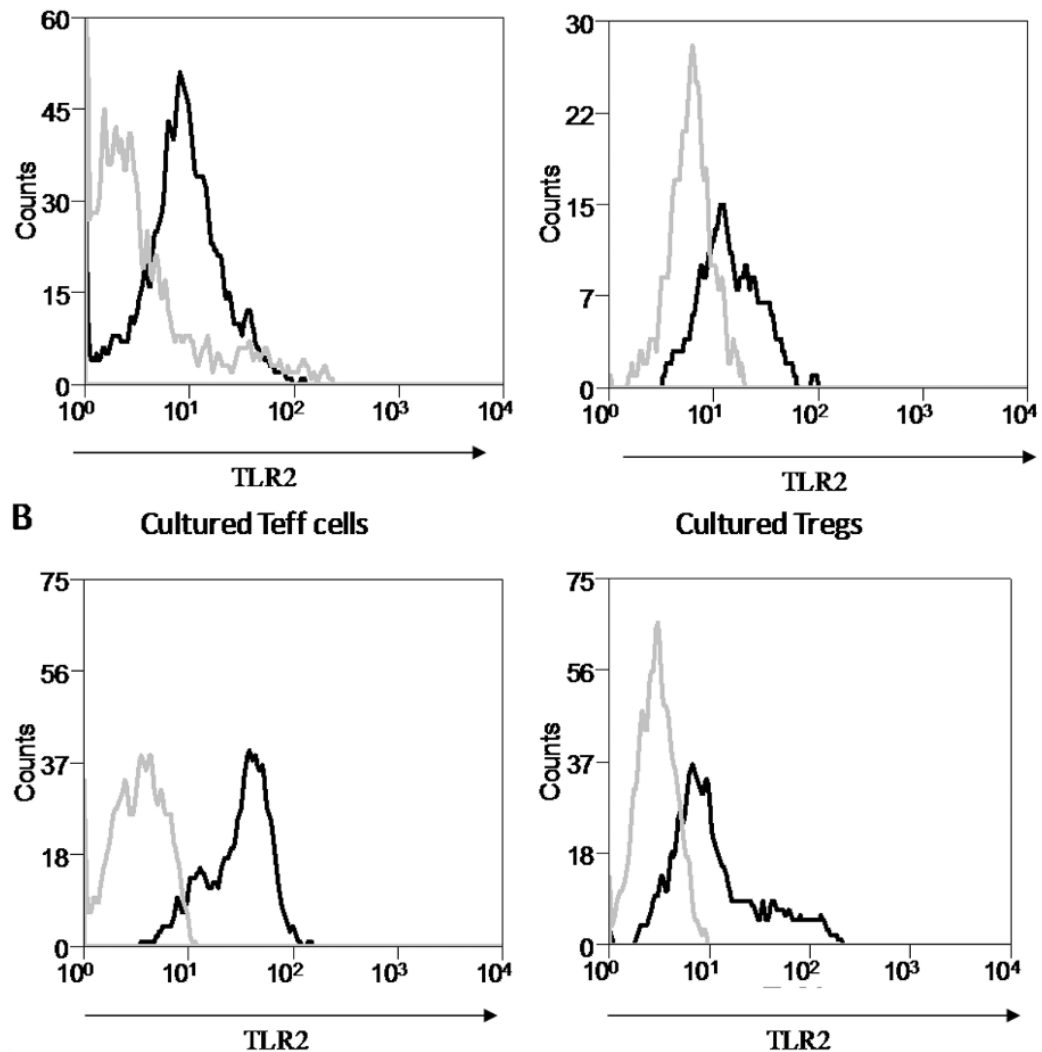

Fresh spleen-isolated naive Teff

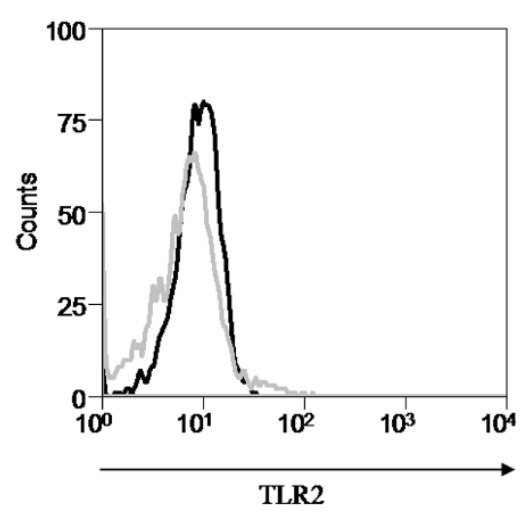

Figure 1 TLR2 expression profile on fresh and cultured immune cells. (A) Dendritic cells $\left(C D 11 c^{+}\right), C D^{+} C D 25^{+}$Foxp3 $3^{+}$Treg and $C D 4^{+}$ effector T cells (Foxp3-) were isolated from spleens of naïve mice and immediately analyzed for TLR2 surface expression by flow cytometry (grey line, isotype control; black line, TLR2 antibody). TLR2 is expressed on freshly isolated DCs, while expression on Teff and Treg is absent or low. (B) Treg and Teff were cultured in the presence of Pam3Cys, anti-CD3 and irradiated splenocytes as APCs for several weeks (see materials and methods). Activation induced TLR2 expression on both Treg and Teff which remained high when cultured for several weeks. Representative results of 3 independent experiments are shown, all experiments were performed in duplicate.

Pam3Cys to these Teff cells did not significantly affect cytokine production.

Analysis of Pam3Cys-stimulated DCs indicated that they readily produce multiple cytokines, including high amounts of IL-6 and IL-12p40 (Figure 4). Maximal cytokine production by DCs already reaches a plateau at relatively low amounts of Pam3Cys $(0.1 \mu \mathrm{g} / \mathrm{ml})$. Overall, the TLR2-induced cytokine profiles of the freshly isolated Teff cells and the DCs nicely correlate with a cytokine profile needed for the induction of an immune response [19-22]. Collectively, these results show that Tregs, DCs as well as Teffs can express functional TLR2. DCs are most capable of sensing low amounts of TLR2 ligand. In agreement with the current understanding that activated Teff cells are less dependent of (co-)stimulation, we show that they are less sensitive to TLR2 co-stimulation than naive Teff. In contrast, Treg remain relatively unresponsive to TCR stimulation and rely on co-stimulation in order to enter a proliferative state.

\section{Effect of TLR2 triggering on Treg function}

Recently, we as well as others reported that triggering of TLR2 on murine Tregs with a relatively high dose of Pam3Cys in combination with TCR signaling induced significant proliferation in the otherwise non-proliferative Treg subset $[14,18]$. Strikingly, Treg proliferation was accompanied by a temporal loss of the ability of the Tregs to suppress the proliferation of the Teff cells. To analyze the effects of TLR2 on Treg function in more detail, we titrated increasing concentrations of the TLR2ligand Pam3Cys in a suppression assay and determined its impact on Teff cell proliferation and on cytokine production, using CFSE-labeled TLR2-deficient Teff cells co-cultured with wildtype Tregs. In contrast to $3 \mathrm{H}-$ tritium-Thymidine incorporation assay, the use of CFSElabeled Teff cells allows to distinguish between Treg and Teff proliferation. As shown in Figure 5A (representative raw data are shown in Additional file 1), very low TLR2ligand concentrations $(<0.2 \mu \mathrm{g} / \mathrm{ml})$ hardly affected the 
Cultured Teffs

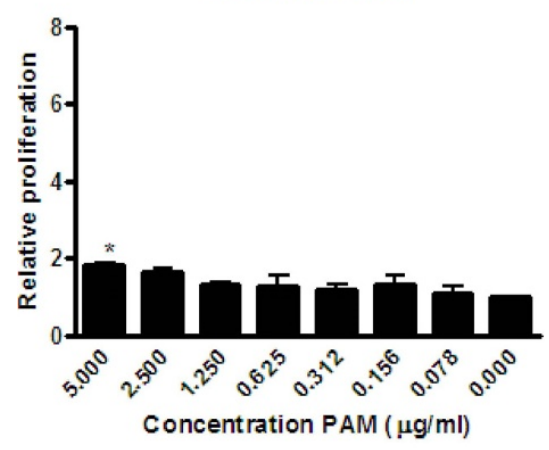

Cultured Tregs

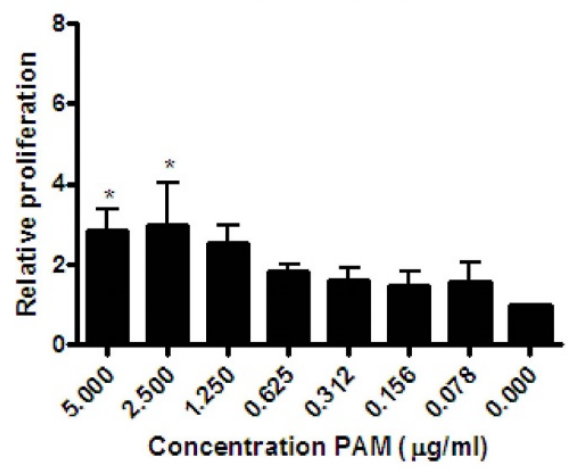

Fresh Teffs

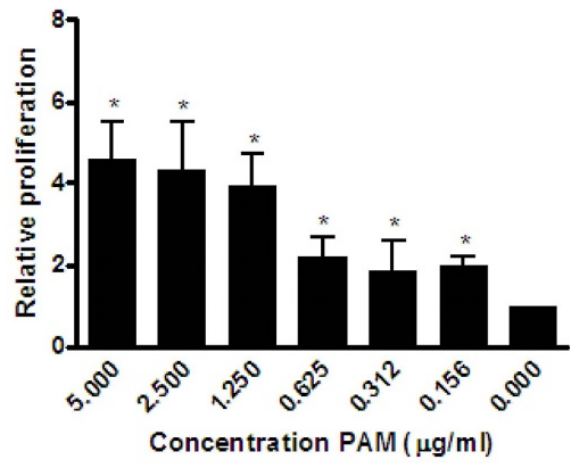

Fresh Tregs

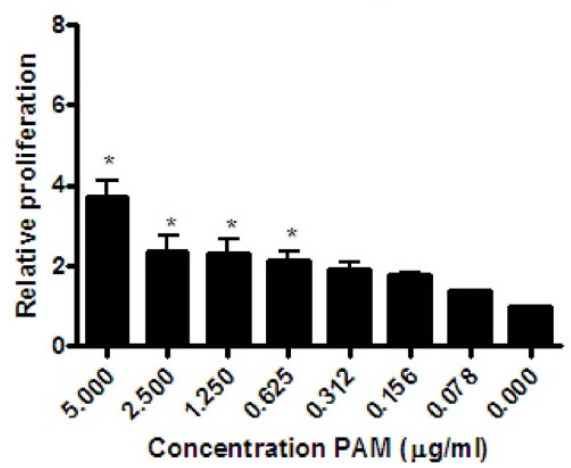

Figure 2 Effect of Pam3Cys titration on proliferation of fresh versus cultured Tregs and Teff cells. Teff and Treg were freshly isolated from spleen or cultured for weeks and stimulated with different concentrations of Pam3Cys in the presence of $1 \mu \mathrm{g} / \mathrm{ml}$ anti-CD3. After 4 days proliferation was determined using thymidine incorporation (relative expression compared to anti-CD3 alone). The condition without Pam3Cys is set to 1 and the conditions with Pam3Cys are related to this condition. The absolute cpm levels of the cells with $(5 \mathrm{ug} / \mathrm{ml})$ and without Pam3Cys are for cultured Teffs 13383 cpm vs 7456 cpm, for fresh Teffs 31076 cpm vs 6693 cpm, for fresh Treg 15590 cpm vs 3776 cpm, and cultured Treg $16642 \mathrm{cpm}$ vs $4936 \mathrm{cpm}$. Freshly isolated Tregs and Teff cells showed increased proliferation upon TLR2 stimulation, while only cultured Tregs (not cultured Teff) remained sensitive to TLR2 triggering. Representative results of 3 independent experiments are shown. The error bars represent standard deviations of triplicate measurements.

Treg mediated suppression of Teff proliferation. In line with our previous results, a higher dose of TLR2-ligands $(>2 \mu \mathrm{g} / \mathrm{ml})$ induced a clear loss of Treg suppressive activity as reflected by increased Teff proliferation. The addition of Tregs to the Teff cell cultures blocked proliferation as well as cytokine production. In contrast to its effect on proliferative capacity, the addition of Pam3Cys to these cultures failed to restore cytokine production by Teffs (Figure 5B). These results indicate that with respect to Treg mediated suppression of Teff cells, suppression of proliferation and suppression of cytokine production should be regarded as two separate events.

Effect of TLR2 triggering on immune cell subsets on Treg mediated immune suppression

To determine the contribution of TLR2 triggering on the different cell types present in a suppression assay (Tregs, Teffs and APCs), we performed suppression assays combining cells isolated from TLR2-deficient mice and wildtype mice. This setup ensures that any of the observed effect of the TLR2-ligands is mediated through TLR2 triggering on the cells purified from the wildtype mice. We focused on Treg-mediated suppression of Teff proliferation as the read out system.

TLR2 triggering on wildtype Tregs in the presence of TLR2-deficient Teff and APC resulted in a $57 \%$ reduction in the suppression of Teff cell proliferation (Figure 6A). Similarly, TLR2-signaling on Teffs (TLR2-deficient Tregs and DCs) or DC (TLR2-deficient Teff and Treg) inhibited Treg-mediated suppression to 55\% and 65\% respectively. A similar level of reduction in suppression was also observed when all cells were derived from wildtype origin. Control suppression assays performed with only TLR2-deficient cells demonstrated the specificity of the TLR2-effect (Figure 6B). These data thus demonstrate that TLR2 is important in controlling activation 


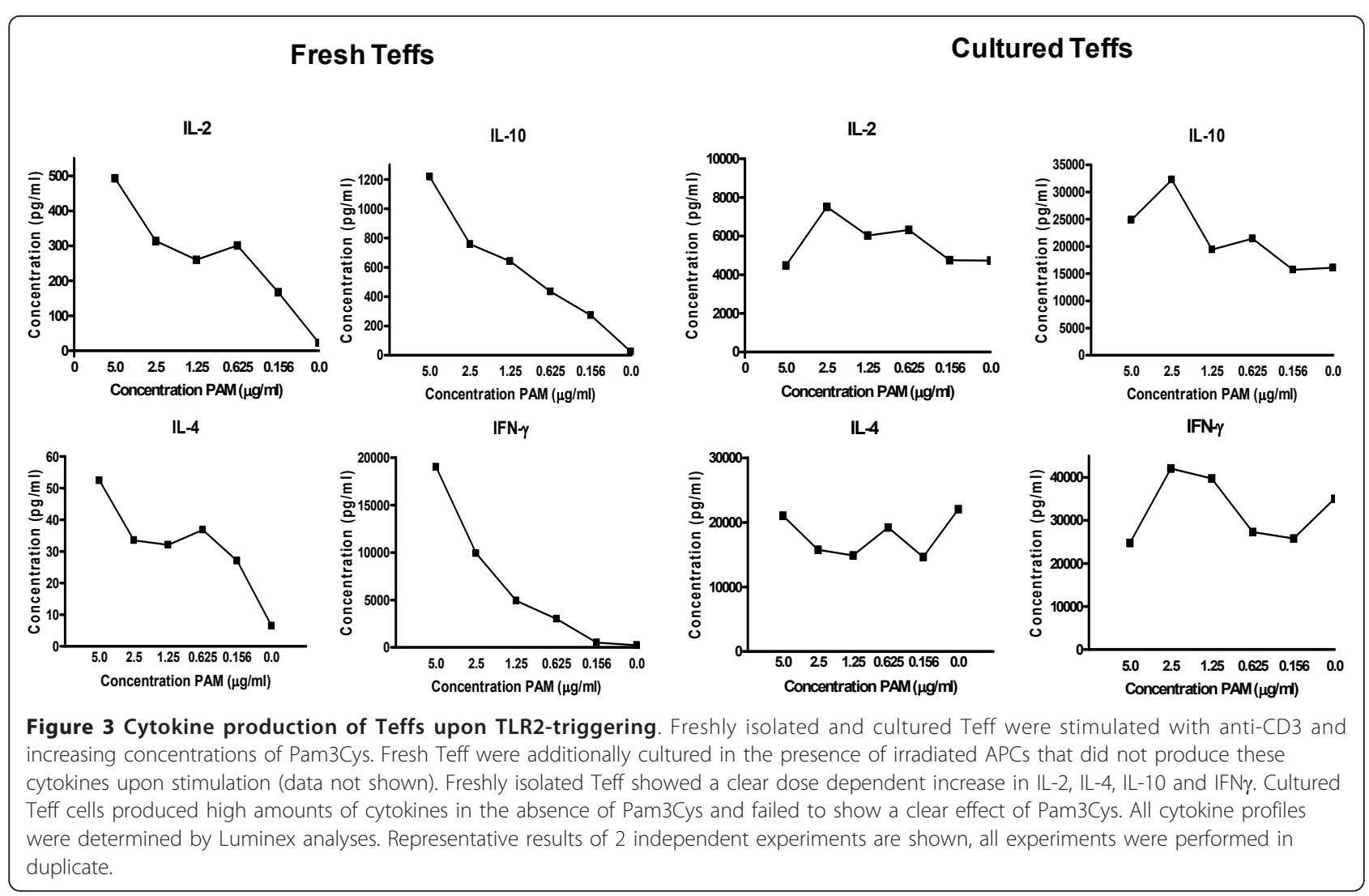

of immune cells and that TLR2 acts on each of the three cell types present in the suppression assay; Tregs, Teffs and APCs.

\section{Effect of Legionella pneumophila on immune cell proliferation, cytokine production and Treg suppression} So far, the experiments were performed with the synthetic TLR2 ligand Pam3Cys. We repeated these experiments with the natural pathogen Legionella pneumophila, which is known to contain high amounts of TLR2 ligands [23]. As shown in Figure 7, the proliferative capacity of the cultured Teff cells and Tregs were similar to the data found with Pam3Cys. The cultured Teff cells were hardly responsive to the HKLP, and this did not change upon increasing pathogen concentration. The naive CD4+ Teff cells were highly responsive to HKLP in a dose-dependent manner. Both freshly-isolated and cultured Teff produced IFN- $\gamma$ upon HKLP stimulation (Figure 8).

To further investigate the effect of HKLP on Treg, suppression assays were performed. The results shown in Figure 9 indicate that HKLP abrogated the Tregmediated suppressive capacity even in conditions where Tregs are the only TLR2 expressing cells. These data imply that the immune modulatory effect of Pam3Cys can be mimicked by a natural pathogen containing high amounts of TLR2 ligand. It is likely that during normal infections of Legionella pneumophila the suppressive capacity of the Tregs can at least in part be abrogated by directly acting on the Tregs themselves.

\section{Discussion}

The complexity of signals received and produced by our immune system has puzzled immunologist for many years. The re-discovery of the suppressor $\mathrm{T}$ cells mostly indicated as Tregs has increased our comprehension of immunity in health and disease. In immune homeostasis, immune activation events are kept in balance by many regulatory mechanisms like suppressors of cytokine signaling (SOCS), CTLA-4, suppressive cytokines like IL-10 and TGF- $\beta$, and importantly Tregs. However during infections, strong immune activation signals are released, including a set of pathogen-associated molecular patterns. These pathogen derived determinants are able to induce the activation of amongst others TLRs, one of the main families of receptors involved in pattern recognition. This will result in a strong inflammatory immune response capable of dealing with the pathogenic challenge. More recently, TLR agonists have been shown to also act directly on Treg and 'lift' the suppressive pressure on other immune cells. We demonstrated that TLR2 triggering on Tregs in combination with TCR signaling induces proliferation of the Tregs and reduces 


\section{DCs}

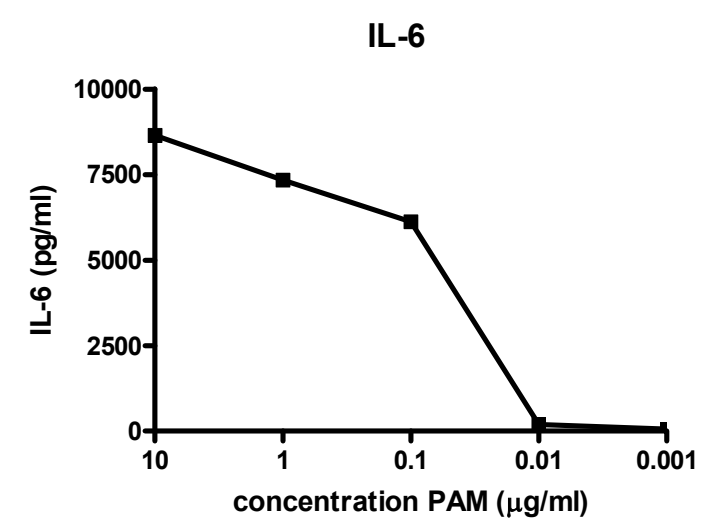

IL-10

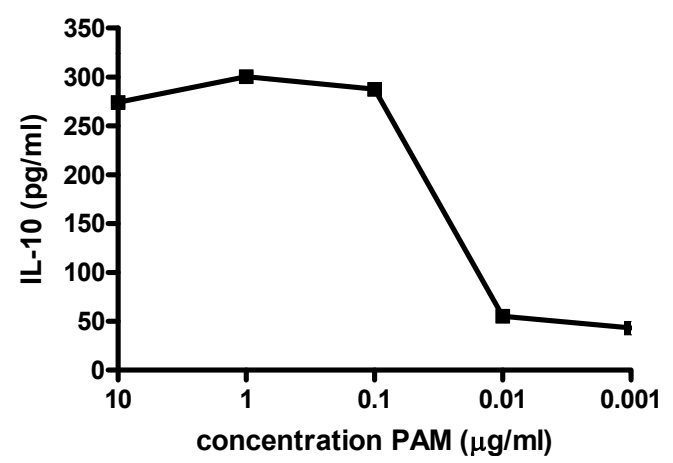

IL-12p40

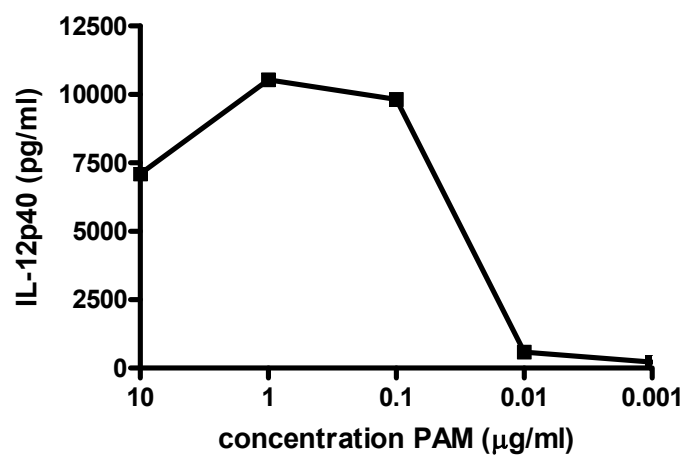

IL-8

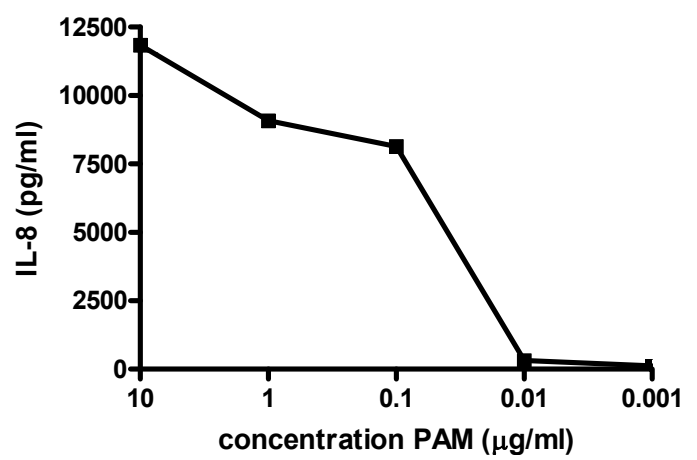

Figure 4 Cytokine production of DCs upon TLR2-triggering. Production of IL-6, IL-12, IL-10 and IL-8 by DCs after stimulation with Pam3Cys. All cytokine profiles were determined by Luminex analyses. Representative results of 2 independent experiments are shown, all experiments were performed in duplicate.

their suppressive capacity [14]. Similar data were later reported by Liu et al. [18]. Recently Oberg et al. [24], examined the influence of different TLR2 ligands on human Tregs. They show similar results for $75 \%$ of the used human donors, namely direct abrogation of the Treg suppressive capacity. They also observed individual differences in efficacy between different TLR2 ligands. Above all, Zhang et al. [25] currently tested the abrogation of Treg-mediated suppression in an in vivo tumorbearing mouse model. They report that injection of the mice in combination with pretreatment of the Treg cells with Pam3Cys, induces a marked reduction of Treg cells in the lymph nodes and a diminished suppressive activity of the remaining Tregs present in these mice. On the contrary, another researcher group has published that TLR2 does not abrogate the Treg suppressive function [26]. The different findings might be explained by the difference in model systems, cell sources, experimental setups and as described by Oberg et al. [24] the expression pattern of TLR1/TLR2.
The loss of suppression by TLR2 triggering on Treg facilitates the induction of adaptive immune responses. At the same time, TLR2-stimulated Tregs expand and have been suggested to limit excessive tissue pathology and to prevent potential autoimmune events once the pathogen is efficiently cleared. To further assess the role of TLR2, we investigated the effects of TLR2 stimulation on Treg and other immune cells known to be involved in the adaptive immune response; Teff cells and the professional antigen presenting DCs. Freshly isolated splenic DCs express TLR2, but the freshly isolated Tregs and Teff cells only express TLR2 upon activation. Interestingly, all freshly isolated cells respond to the TLR2ligand Pam3Cys, however after in vitro culturing, only the Tregs remained responsive to TLR2-stimulation in lower concentrations. TLR2 on naive Teff cells enhanced Teff cell proliferation indicating that TLR2 acts as a co-stimulatory signal, similar to CD28. Cultured Teff cells did not seem to respond to TLR2 triggering. These data are in line with the idea that primed 


\section{A}

\section{Influence PAM on Treg suppression}

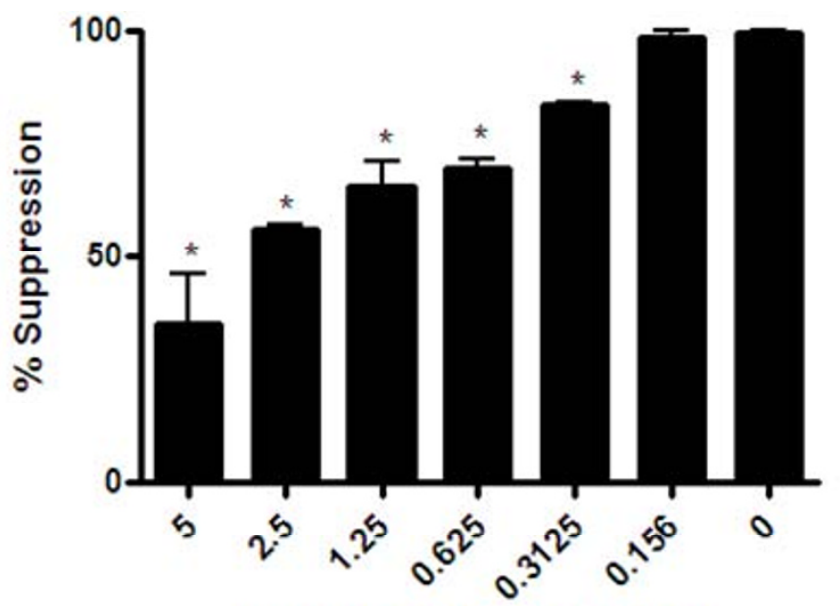

Concentration PAM $(\mu \mathrm{g} / \mathrm{ml})$

B

IL-10

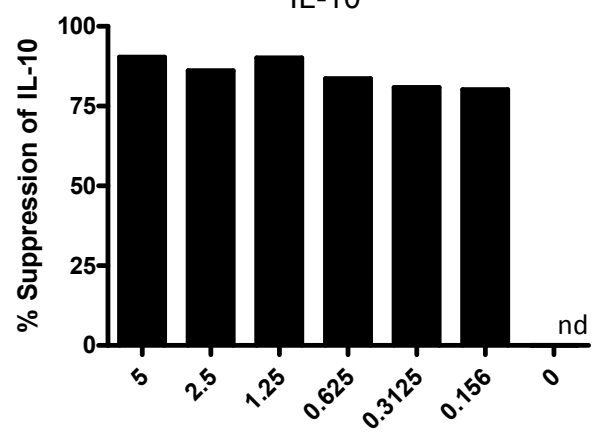

Concentration PAM $(\mu \mathrm{g} / \mathrm{ml})$

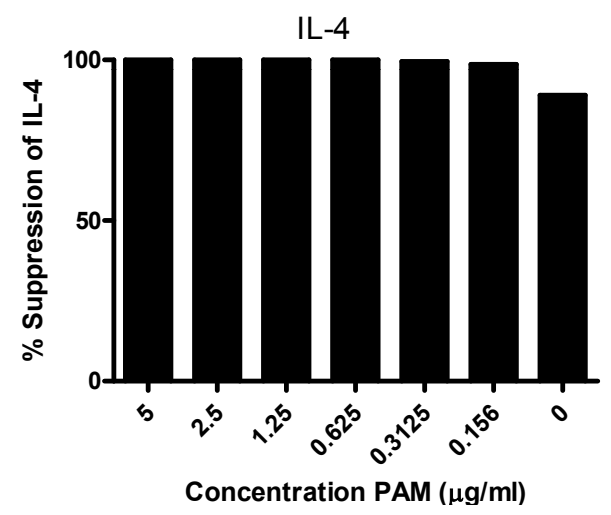

IL-2

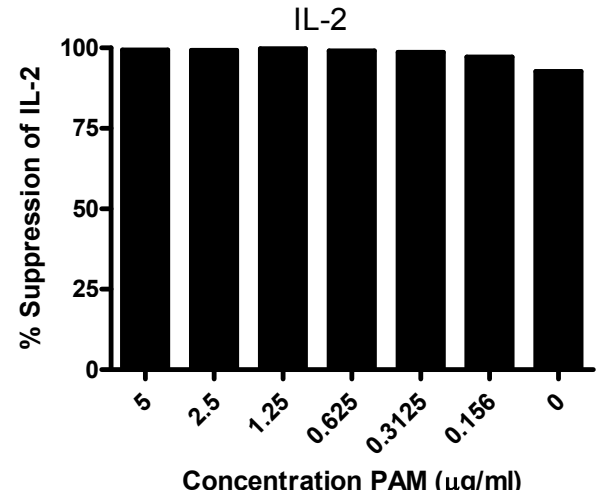

IFN- $\gamma$

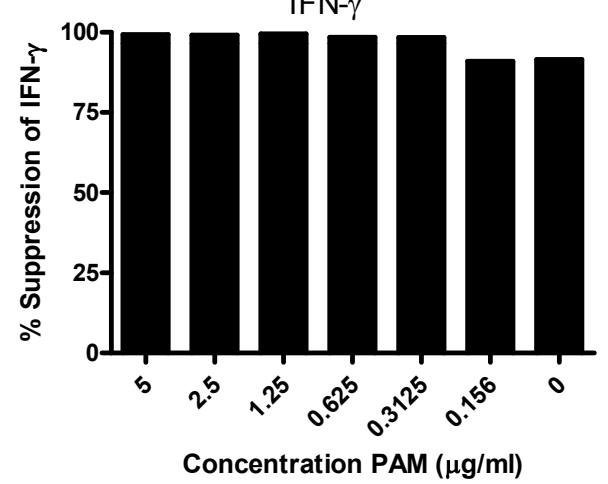

Figure 5 TLR2 triggering effects Treg suppression. (A) Suppression assays were performed with wild type Treg and TLR2-deficient Teff in the absence or presence of Pam3Cys, in which the read-out is Teff cell proliferation. Pam3Cys inhibited Treg-mediated suppression in a dosedependent manner. (B) Analysis of the effect of Pam3Cys on IL-2, IL-4, IL-10 and IFN $\gamma$ cytokine production. Increasing concentrations of Pam3Cys did not restore cytokine production (nd, not detectable). Data representative of 2 experiments are shown. The error bars represent standard deviations of duplicate measurements. 


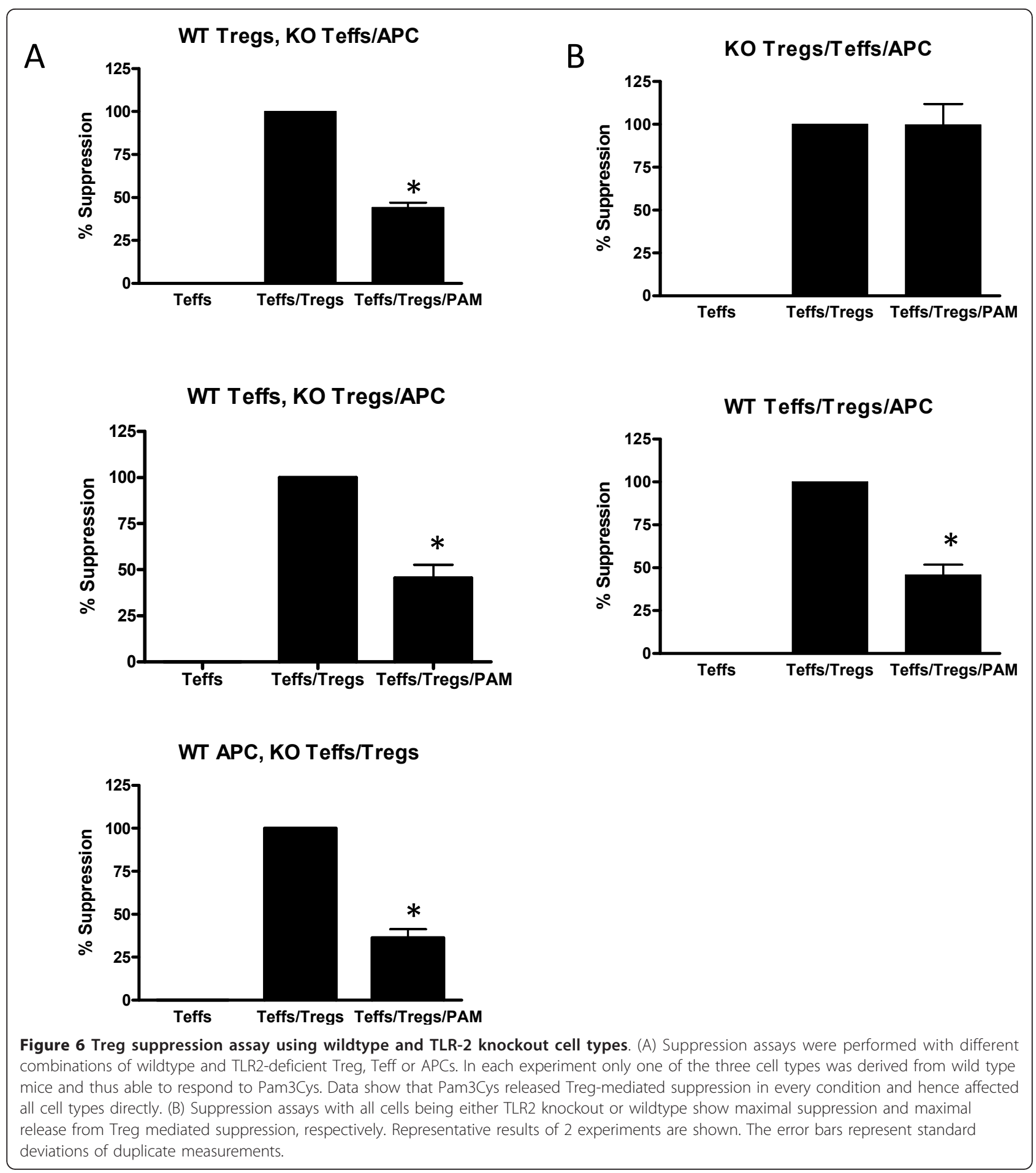

Teff cells are less dependent on co-stimulation by TLR2, similar to co-stimulation by CD28, though the anergic Treg subset requires more than TCR triggering alone in order to enter a state of proliferation.

When analyzing the effects of TLR2-stimulation of Tregs in more detail, we observed a difference between suppression of proliferation and suppression of cytokine production by the Treg. Interestingly, TLR2-treated Treg still suppressed cytokine secretion, but lost their ability to suppress the proliferation of Teff cells. Apparently, the process of suppression of cytokine secretion is different from that of suppression of proliferation. This 


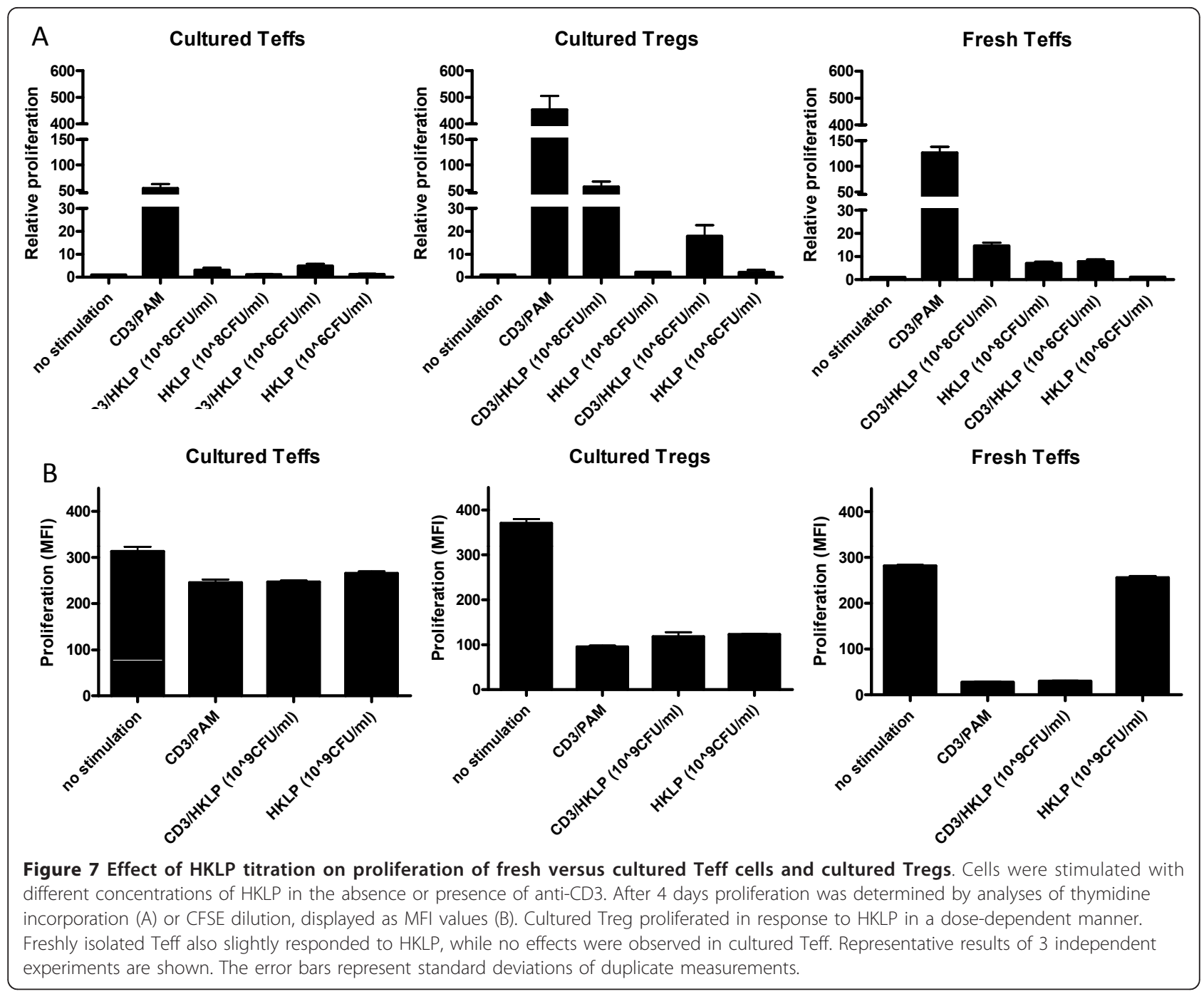

is in line with data of Shevach et al. showing that mature DCs abrogated Treg-mediated suppression of Teff cell proliferation, while cytokine secretion was still suppressed [10]. Moreover, DCs stimulated the proliferation of Treg, similar as observed after TLR2 stimulation. It remains intriguing why such highly different signals (TLR2 versus mature DCs) would yield similar phenotypes in the Tregs. One possibility would be that the Tregs require a certain threshold of co-stimulatory signals. Indeed, it has been shown that TLR2 can substitute for CD28 with respect to co-stimulatory signals required for $\mathrm{T}$ cell stimulation [21]. Once the combination of co-stimulatory signals and TCR triggering is sufficient, the Treg will suppress only cytokine secretion by Teff and will start to proliferate themselves. Why would Treg allow Teff proliferation whilst inhibiting Teff cytokine production? Possibly cytokine secretion is a far more dangerous effector mechanism as compared to Teff proliferation.
Since TLR2 effected suppression of proliferation, we addressed the effects of TLR2 on each of the cellular subsets present during the suppression assay. Our results show that TLR2 can abrogate the suppression of proliferation by acting on each subset being Treg, Teff or DC. TLR2 triggering on Treg induces their proliferation and temporal inhibition of their suppressive capacity, while on Teff cells TLR2 has a co-stimulatory role. TLR2 triggering on DCs results in the production of cytokines like IL-6 that have been shown to release Teff from Treg mediated suppression [27]. We examined IL6 production in our co-culture experiments combining Teffs, Tregs and DCs for wild type and TLR2-knock out origin (data not shown). Although the presence of IL-6 coincides with the abrogation of Treg-mediated suppression of Teff proliferation in the wild type conditions, Teffs also start to proliferate when Teffs/DCs are TLR2deficient. We therefore conclude that IL-6 is able to stimulate the proliferation of Teffs but is not absolutely 
Fresh Tefis

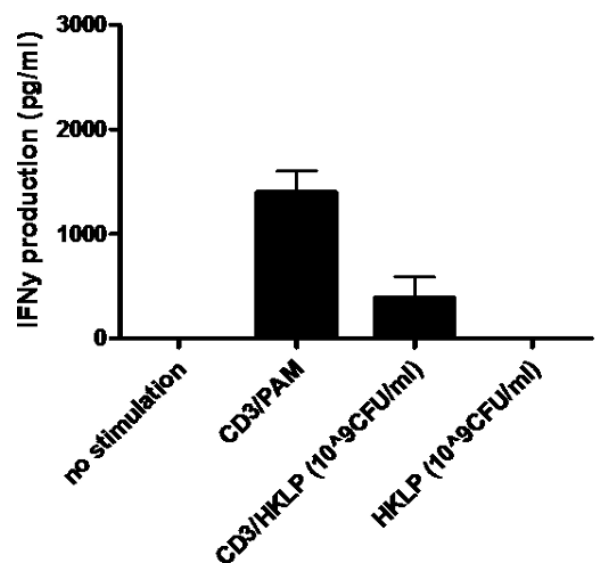

Cultured Tefis

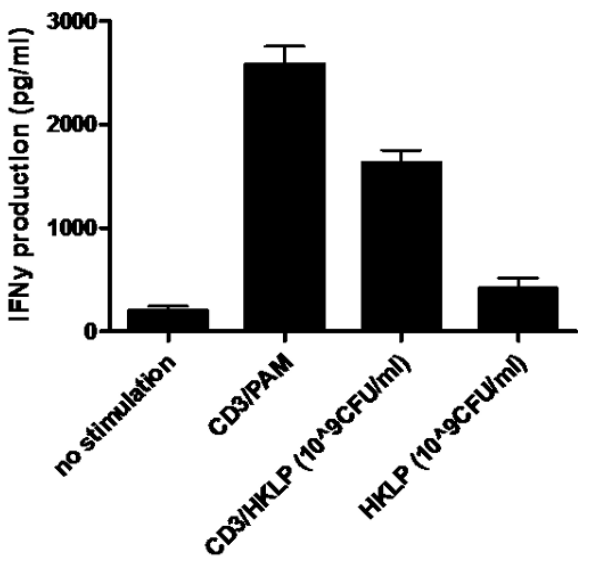

Figure 8 IFN $\gamma$ cytokine production by HKLP stimulation. Cultured Teff cells and freshly isolated Teff cells were stimulated with $10 \wedge 9$ CFU/ml HKLP in the absence or presence of anti-CD3. Representative results of 2 independent experiments are shown. The error bars represent standard deviations of duplicate measurements.

required for abrogation of Treg-mediated suppression of Teff proliferation. By acting on all multiple immune cell subsets involved in adaptive immunity, TLR2 stimulation launches an effective immune response. A potent increase of regulatory mechanisms will have to occur to suppress any potential autoimmune events. The observed expansion of Tregs following immune activation shown here and in our previous work represents one of these feed-back mechanisms. The increased expansion of Treg may prevent immune pathology and help to dampen the immune response once the cause of the immune challenge has been eliminated.
The question arose whether the effect of synthetic TLR2 ligand could also be observed during natural infections. The gram-negative micro-organism Legionella pneumophila is the causing agent of a severe pneumonia. Immune recognition of this microorganism has been reported to be dependent on TLR2 signaling and not on TLR4 signaling $[15,16]$. Therefore we repeated the experiments with heat-killed Legionella pneumophila (HKLP) to confirm our Pam3Cys findings. Indeed the effect of TLR2 triggering on all different immune cells could be confirmed when using HKLP in our assays. Most importantly the suppressive capacity of

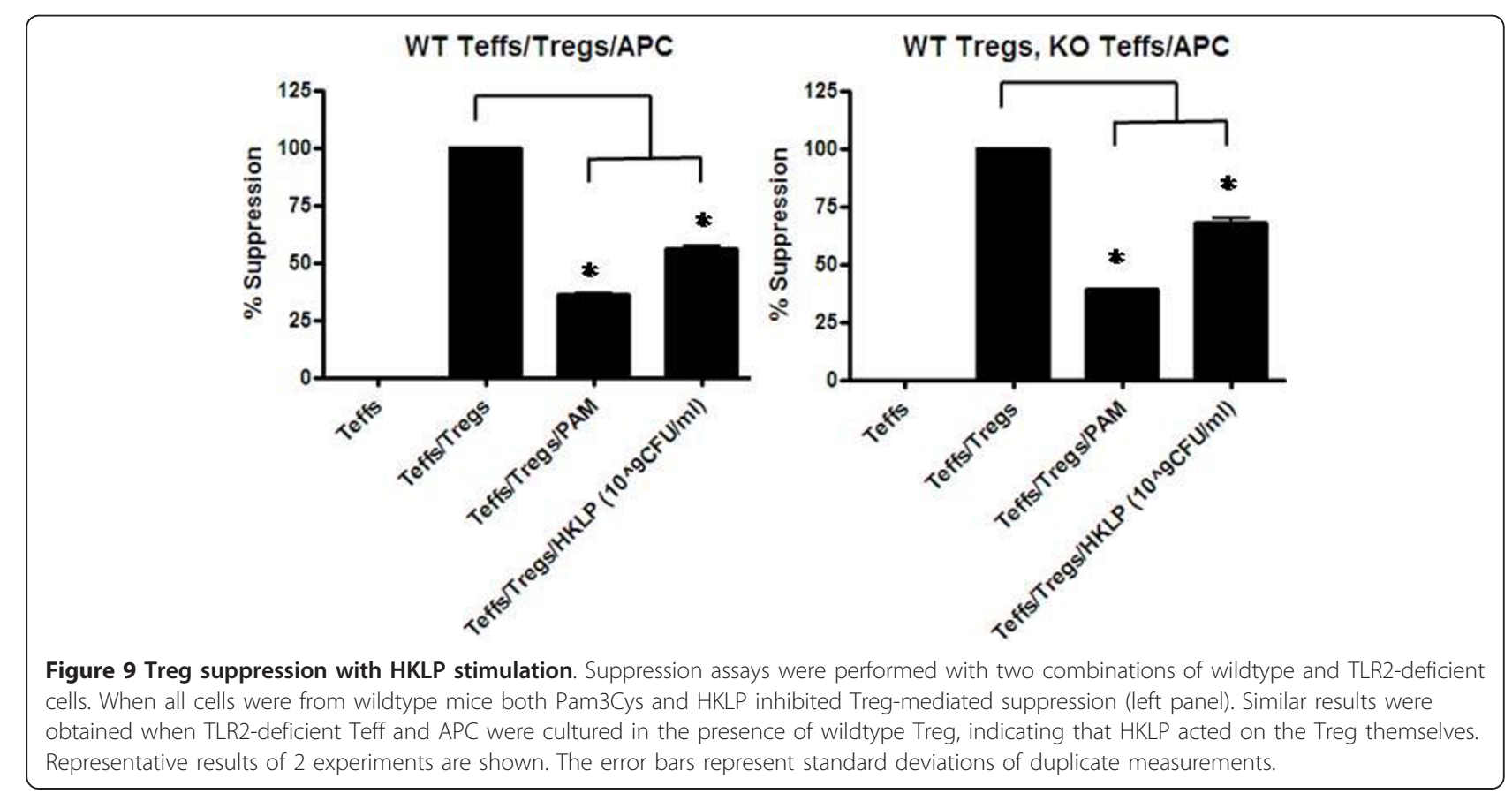


the Tregs could be abrogated in the presence of HKLP. These data indicate that TLR2 triggering potently modulates immune function during bacterial infections. However, it should be considered that besides effects on TLR2, stimulation of other pathogen recognition receptors could drastically influence the outcome of induced immune responses against specific pathogens. Recognition of Legionella pneumophila is mainly relying on TLR2 triggering. Other pathogens likely trigger other receptors that could possibly effect Treg suppression.

\section{Conclusions}

Our findings indicate that Treg-mediated suppression of $\mathrm{T}$ helper cell proliferation is functionally distinct from suppression of cytokine secretion. TLR2 acts on multiple cells of the adaptive and innate immune system thereby providing direct immune stimulation of Teffs and DCs while simultaneously inhibiting the suppressive abilities of Tregs. These effects are not only observed for a synthetic TLR2 ligand, but could be confirmed with the natural TLR2 ligand containing bacteria Legionella pneumophilia. Further understanding of the precise mechanisms involved, will most likely contribute to new strategies for immune-based intervention strategies.

\section{Methods \\ Mice}

The wildtype C57BL/6 mice were obtained from Charles River WIGA (Sulzfeld, Germany) Gmbh. TLR2 knockout on C57BL/ 6 background mice were kindly obtained from S. Akira (Osaka University, Osaka, Japan). All animal experiments were approved by the Animal Experimental Committee of Radboud University Nijmegen Medical Centre and were performed in accordance with institutional and (inter)national guidelines.

\section{Antibodies and flow cytometry}

Directly labeled monoclonal antibodies used for staining by anti-CD4-APC or -FITC (clone L3T4), anti-CD25FITC or -PE (clone 7D4), anti-CD11c-FITC (HL3), antiStreptavidin-PerCp, and their isotype controls were obtained from BD Biosciences - Pharmingen. AntimTLR2-biotine (clone T2.5), anti-mFoxP3-biotine (clone FJK-16S) and the isotype control were obtained from eBioscience. Analysis of cell surface markers on lymphocytes was performed using FACScalibur (BD) and CELLQuest software (version 3.3; BD Biosciences Pharmingen).

\section{T cell purification and analysis}

Spleens from wildtype or TLR2 knockout mice were mashed and filtered, and CD4+ T cells were purified using anti-mouse-CD4 Microbeads (MACS, Miltenyi Biotec), resulting in a enriched $\mathrm{CD} 3+\mathrm{CD} 4+\mathrm{T}$ cell population. Naive CD4+CD25low Teff cells and naive CD4+CD25high Treg cell subsets were obtained by flow cytometry purification of the pre-sorted CD4+ T cells; CD4 cells were stained with FITC-conjugated CD4 mAb (BD bioscience, clone L3T4) and PE-conjugated CD25 (BD biosciences, clone 7D4). Cell sorting was performed on a Coulter Altra HyPerSort cell sorter. Both naive CD4+CD25low $\mathrm{T}$ cells and naive CD4+CD25high $\mathrm{T}$ cells were $98 \%$ pure, based on CD25 expression pattern. Determining the purity by using biotinilated-FoxP3 Ab (eBiosciences, clone FJK-16S) and streptavidinePerCp Ab (BD bioscience) showed that from the CD25high FACSsorted cells, 67\% of the cells were FoxP3+, therefore Tregs. Sorted cells were used directly in several assays or kept in culture as described below. After several weeks of culture the purity of the Treg cell line as well as the Teff cell line (referred in this paper to the cultured Tregs or cultured Teff cells) was $96 \%$ or higher (see Additional file 2).

\section{Treg and Teff cell culture and suppression assay}

FACS-sorted purified CD4+CD25+ T cells and CD4 + CD25-T cells are kept in culture for several weeks. Each cell-line is cultured in $10^{\wedge} 4$ cells per well of a 96 well plate, and were stimulated weekly with $5^{*} 10^{\wedge} 4$ irradiated CD4-MACS bead depleted splenocytes per well, in $2 \mu \mathrm{g} / \mathrm{ml}$ Pam3Cys (EMC microcollections, Germany), $1 \mu \mathrm{g} / \mathrm{ml}$ anti-CD3 (145-2C11; BD Biosciences - Pharmingen), and $120 \mathrm{IU}$ (international units) IL-2/ml complete medium. The cells were washed 3 days after each stimulation and maintained in culture medium supplemented with 120 IU IL-2/ml. When necessary, dead cells were removed by ficoll density gradient. Cultured Tregs or Teff cells were used in assays at least 6 days after stimulation, in this case the Tregs and Teff cells are in a resting state when used in all assays.

Suppression assays were performed as follows; freshly sorted (wildtype or TLR2 knockout) CD4+CD25-naive $\mathrm{T}$ cells $\left(20^{*} 10^{\wedge} 3\right.$ per well) and either cultured or freshly isolated (wildtype or TLR 2 knockout) Tregs $\left(20^{*} 10^{\wedge} 3\right.$ per well) were mixed and cocultured for 3 days with $20 * 10^{\wedge} 3$ irradiated (wildtype or TLR2 knockout) APCs per well. If indicated, the $\mathrm{T}$ cells were stimulated with TLR ligand Pam3Cys $(5 \mu \mathrm{g} / \mathrm{ml}$, EMC microcollections $\mathrm{GmbH})$ or HKLP $\left(10^{\wedge} 6-10^{\wedge} 9 \mathrm{CFU} / \mathrm{ml}\right.$ of heat-killed Legionella pneumophila; InvivoGen) with or without soluble anti-CD3 (1 $\mu \mathrm{g} / \mathrm{ml}, 145-2 \mathrm{C} 11$; BD Biosciences Pharmingen) in complete medium. After 3 days of coculture, supernatant was collected for cytokine analysis. In addition, the suppression of proliferation was monitored by analyzing the CFSE-labeled $(1 \mu \mathrm{M})$ freshly sorted (wildtype or TLR2 knockout) CD4+CD25-naive $\mathrm{T}$ cells. CFSE fluorescence intensity was measured by flow cytometry. 


\section{Calculation of suppression}

The amount of suppression is calculated by the amount of proliferation or cytokine production. In each assay we include 5 conditions (see Additional file 3) by which we calculate the suppression, using the mean fluorescent intensity of the CFSE-labeled Teff cells. The first condition (background) is a coculture of CFSE-labeled Teff cells, APCs and anti-CD3 stimulation $(1 \mu \mathrm{g} / \mathrm{ml})$. This condition indicates the level of Teff cell proliferation induced by anti-CD3 in the presence of APC. The second condition (0\% suppression) is a coculture of CFSE-labeled Teff cells, APCs, anti-CD3 stimulation $(1 \mu \mathrm{g} / \mathrm{ml})$ and Pam3Cys (concentration used between 10 to $0.01 \mu \mathrm{g} / \mathrm{ml}$ ). This condition represents maximum of Teff cell proliferation. The next condition consists of a coculture of CFSE-labeled Teff cells, APCs, Tregs and anti-CD3 stimulation $(1 \mu \mathrm{g} / \mathrm{ml})$, which is used to achieve maximal suppression (set to $100 \%$ as a reference). The last condition consists of a coculture of CFSE-labeled Teff cells, APCs, Tregs, anti-CD3 stimulation $(1 \mu \mathrm{g} / \mathrm{ml})$ and Pam3Cys (concentration used between 10 to 0.01 $\mu \mathrm{g} / \mathrm{ml})$, indicated as ' $x \%$ suppression'. This $x \%$ suppression is calculated by the following formula; ((MFI x\% - MFI 0\%)*100)/(MFI max - MFI 0\%). For all data presented the difference between the $0 \%$ suppression and max suppression was at least 150 in MFI. In the case of cytokine production, we used the same formula with amount of cytokines produced as principle parameter.

\section{Cytokine measurements}

For the detection of cytokines in culture supernatant we used the Mouse Th1/Th2 Bio-Plex Cytokine Assay (\#171-F11081, Bio-Rad, Hercules, CA). All procedures were performed according to the manufacturer's instructions.

\section{Proliferation assay}

Freshly FACS-sorted or cultured CD4+CD25+Treg and CD4+Tconv cells were cultured for 4 days with a range of Pam3Cys concentrations (between $0 \mu \mathrm{g} / \mathrm{ml}-5 \mu \mathrm{g} / \mathrm{ml}$ ) or HKLP $\left(10^{\wedge} 6-10^{\wedge} 9 \mathrm{CFU} / \mathrm{ml}\right.$ of heat-killed Legionella pneumophila; InvivoGen) with or without soluble CD3 stimulation $(1 \mu \mathrm{g} / \mathrm{ml})$. After 4 days the proliferation was measured by overnight ( 20 hours) thymidine incorporation.

\section{Statistical analysis}

The data are analysed using a one-way ANOVA test, to test the differences between groups (PRISM software version 4.0; GraphPad, San Diego, CA, USA). Statistical significance is inferred at $\mathrm{P}<0.05$. Significant differences are indicated with an asterisks $\left({ }^{*}\right)$.

\section{Additional material}

Additional file 1: Suppression assay with increasing Pam3Cys concentrations. Suppression is determined by CFSE dilution of the naive Teff cells. These data show the CFSE peaks obtained after 3-4 days culture with or without Pam3Cys $(5 \mu \mathrm{g} / \mathrm{ml})$. Loss of Treg-mediated suppression resulted in more Teff proliferation, indicated by a peak shift to the left.

Additional file 2: Purity of isolated Tregs and Teffs. The Teff cells and Tregs were MACS-sorted from total splenocytes based on CD4 expression and thereafter FACS-sorted into CD25 high or low expressing cells. After several weeks in culture, the purity of Teff and Treg was 98\% and $96 \%$ respectively.

Additional file 3: Conditions used in suppression assay. This list shows all conditions always used in suppression assays, and which cells/ stimulations are in each condition.

\section{List of abbreviations}

APC: antigen presenting cell; DC: dendritic cell; FoxP3: fork-head box protein 3; HKLP: heat-killed Legionella pneumophila; HSP60: heat-shock protein 60 IFNY: interferon gamma; IL-...: interleukin...; MFI: mean fluorescent intensity; NLR: NOD like receptor; Pam3Cys: Pam3CysSK4; PAMPs: pathogen associated molecular patterns; RA: rheumatoid arthritis; RLR: RIG-I like receptor; Teff(s): effector T cell(s); TGF $\beta$ : transforming growth factor beta; TLR...: toll like receptor...; Treg(s): regulatory $T$ cell(s).

\section{Acknowledgements}

This work was supported by Top Institute Pharma, within the framework of project D1-101.

\section{Author details}

${ }^{1}$ Department of Tumor Immunology, Nijmegen Centre for Molecular Life Sciences, Radboud University Nijmegen Medical Centre, Nijmegen, the Netherlands. ${ }^{2}$ Schering-Plough Research Institute, Target Discovery Oss, Molenstraat 110, 5340 BH Oss, The Netherlands.

\section{Authors' contributions}

WWCVM contributed to this article by performing most of the experiments. LWT and JMB contributed by mostly doing the cytokine assays, and LWT also performed several suppression assays. SN contributed by giving intellectual input. RPMS and GJA are both equally responsible for the total study concept, study design and guidance. GJA is the daily supervisor and the Principle Investigator. All authors have read and approved the final manuscript.

Received: 14 October 2010 Accepted: 24 March 2011 Published: 24 March 2011

\section{References}

1. Creagh EM, O'Neill LA: TLRs, NLRs and RLRs: a trinity of pathogen sensors that co-operate in innate immunity. Trends Immunol 2006, 27:352-357.

2. Ishii KJ, Akira S: Toll or toll-free adjuvant path toward the optimal vaccine development. J Clin Immunol 2007, 27:363-371.

3. Netea MG, Van der Meer JW, Sutmuller RP, Adema GJ, Kullberg BJ: From the Th1/Th2 paradigm towards a Toll-like receptor/T-helper bias. Antimicrob Agents Chemother 2005, 49:3991-3996.

4. Miyake K: Innate immune sensing of pathogens and danger signals by cell surface Toll-like receptors1. Semin Immunol 2007, 19:3-10.

5. Jin MS, Kim SE, Heo JY, Lee ME, Kim HM, Paik SG, et al: Crystal structure of the TLR1-TLR2 heterodimer induced by binding of a tri-acylated lipopeptide1. Cell 2007, 130:1071-1082.

6. Zahringer U, Lindner B, Inamura S, Heine H, Alexander C: TLR2 promiscuous or specific? A critical re-evaluation of a receptor expressing apparent broad specificity. Immunobiology 2008, 213:205-224.

7. Murciano C, Villamon E, Gozalbo D, Roig P, O'Connor JE, Gil ML: Toll-like receptor 4 defective mice carrying point or null mutations do not show increased susceptibility to Candida albicans in a model of 
hematogenously disseminated infection. Medical Mycology 2006, 44:149-157.

8. Netea MG, Sutmuller R, Hermann C, Van der Graaf CA, Van der Meer JW van Krieken $\mathrm{JH}$, et al: Toll-like receptor 2 suppresses immunity against Candida albicans through induction of IL-10 and regulatory T cells. J Immunol 2004, 172:3712-3718.

9. Abdollahi-Roodsaz S, Joosten LA, Roelofs MF, Radstake TR, Matera G, Popa C, et al: Inhibition of toll-like receptor 4 breaks the inflammatory loop in autoimmune destructive arthritis1. Arthritis Rheum 2007, 56:2957-2967.

10. Shevach EM: Regulatory T cells in autoimmmunity. Annu Rev Immunol 2000, 18:423-449.

11. Hori S, Nomura T, Sakaguchi S: Control of regulatory T cell development by the transcription factor Foxp3. Science 2003, 299:1057-1061.

12. Sakaguchi S, Sakaguchi N, Asano M, Itoh M, Toda M: Immunologic selftolerance maintained by activated T cells expressing $\mathrm{IL}-2$ receptor alphachains (CD25). Breakdown of a single mechanism of self-tolerance causes various autoimmune diseases. J Immunol 1995, 155:1151-1164.

13. Sakaguchi S, Ono M, Setoguchi R, Yagi H, Hori S, Fehervari Z, et al: Foxp3 $+\mathrm{CD} 25+\mathrm{CD} 4+$ natural regulatory $\mathrm{T}$ cells in dominant self-tolerance and autoimmune disease. Immunol Rev 2006, 212:8-27.

14. Sutmuller RP, den Brok MH, Kramer M, Bennink EJ, Toonen LW, Kullberg BJ, et al: Toll-like receptor 2 controls expansion and function of regulatory $T$ cells. J Clin Invest 2006, 116:485-494.

15. Lettinga KD, Florquin $S$, Speelman $P$, van Ketel $R$, van der Poll $T$, Verbon $A$ Toll-like receptor 4 is not involved in host defense against pulmonary Legionella pneumophila infection in a mouse model. J Infect Dis 2002, 186:570-573.

16. Girard R, Pedron T, Uematsu S, Balloy V, Chignard M, Akira S, et al: Lipopolysaccharides from Legionella and Rhizobium stimulate mouse bone marrow granulocytes via Toll-like receptor 2. J Cell Sci 2003, 116:293-302.

17. Schindler U, Baichwal VR: Three NF-kB binding sites in the human Eselectin gene required for maximal tumor necrosis factor alpha-induced expression. Mol Cell Biol 1994, 14:5820-5831

18. Liu H, Komai-Koma M, Xu D, Liew FY: Toll-like receptor 2 signaling modulates the functions of CD4+ CD25+ regulatory T cells. Proc Natl Acad Sci USA 2006, 103:7048-7053.

19. Asai Y, Makimura Y, Ogawa T: Toll-like receptor 2-mediated dendritic cell activation by a Porphyromonas gingivalis synthetic lipopeptide. J Med Microbiol 2007, 56:459-465

20. Kiura K, Kataoka H, Nakata T, Into T, Yasuda M, Akira S, et al: The synthetic analogue of mycoplasmal lipoprotein FSL-1 induces dendritic cell maturation through Toll-like receptor 2. FEMS Immunol Med Microbiol 2006, 46:78-84.

21. Komai-Koma M, Jones L, Ogg GS, Xu D, Liew FY: TLR2 is expressed on activated T cells as a costimulatory receptor. Proc Natl Acad Sci USA 2004, 101:3029-3034

22. Weigt $H$, Muhlradt PF, Emmendorffer A, Krug N, Braun A: Synthetic mycoplasma-derived lipopeptide MALP-2 induces maturation and function of dendritic cells. Immunobiology 2003, 207:223-233.

23. Shim HK, Kim JY, Kim MJ, Sim HS, Park DW, Sohn JW, et al: Legionella lipoprotein activates toll-like receptor 2 and induces cytokine production and expression of costimulatory molecules in peritoneal macrophages. Exp Mol Med 2009, 41:687-694.

24. Oberg HH, Hoa Ly TT, Ussat S, Meyer T, Kabelitz D, Wesch D: Differential but direct abolishment of human regulatory $\mathrm{T}$ cell suppressive capacity by various TLR2 ligands. J Immunol 2010, 184:4733-4740.

25. Zhang Y, Luo F, Cai Y, Liu N, Wang L, Xu D, et al: TLR1/TLR2 agonist induces tumor regression by reciprocal modulation of effector and regulatory T cells. J Immunol 2011, prepublished online 7 January 2011.

26. Chen Q, Davidson TS, Huter EN, Shevach EM: Engagement of TLR2 does not reverse the suppressor function of mouse regulatory $T$ cells, but promotes their survival. J Immunol 2009, 183:4458-4466.

27. Pasare C, Medzhitov R: Toll pathway-dependent blockade of CD4+CD25+ T cell-mediated suppression by dendritic cells. Science 2003, 299:1033-1036.

doi:10.1186/1471-2172-12-23

Cite this article as: van Maren et al:: Multifaceted effects of synthetic TLR2 ligand and Legionella pneumophilia on Treg-mediated suppression of T cell activation. BMC Immunology 2011 12:23.

\section{Submit your next manuscript to BioMed Central and take full advantage of:}

- Convenient online submission

- Thorough peer review

- No space constraints or color figure charges

- Immediate publication on acceptance

- Inclusion in PubMed, CAS, Scopus and Google Scholar

- Research which is freely available for redistribution

Submit your manuscript at www.biomedcentral.com/submit
Biomed Central 